typeset using JPSJ.sty $<$ ver.1.0b $>$

\title{
Coupled CDW and SDW Fluctuations as an Origin of Anomalous Properties of Ferromagnetic Superconductor $\mathrm{UGe}_{2}$
}

\author{
Shinji Watanabe* and Kazumasa Miyake \\ Department of Physical Science, Osaka University, Toyonaka, 560-8531, Japan
}

(Received November 10, 2018)

\begin{abstract}
It is shown that anomalous properties of $\mathrm{UGe}_{2}$ can be understood in a unified way on the basis of a single assumption that the superconductivity is mediated by the coupled SDW and CDW fluctuations induced by the imperfect nesting of the Fermi surface with majority spins at $T=T_{\mathrm{x}}(P)$ deep in the ferromagnetic phase. Excess growth of uniform magnetization is shown to develop in the temperature range $T<T_{\mathrm{x}}(P)$ as a mode-coupling effect of coupled growth of SDW and CDW orderings, which has been observed by two different types of experiments. The coupled CDW and SDW fluctuations are shown to be essentially ferromagnetic spin fluctuations which induce a spin-triplet p-wave attraction. These fluctuations consist of two modes, spin and charge fluctuations with large momentum transfer of the nesting vector. An anomalous temperature dependence of the upper critical field $H_{\mathrm{c} 2}(T)$ such as crossing of $H_{\mathrm{c} 2}(T)$ at $P=11.4$ kbar and $P=13.5 \mathrm{kbar}$, can be understood by the strong-coupling-superconductivity formalism. Temperature dependence of the lattice specific heat including a large shoulder near $T_{\mathrm{x}}$ is also explained quite well as an effect of a kind of Kohn anomaly associated with coupled CDW-SDW transition.
\end{abstract}

KEYWORDS: ferromagnetic superconductor, coupled SDW and CDW fluctuations, $\mathrm{UGe}_{2}$

\section{$\S 1 . \quad$ INTRODUCTION}

The recent finding of the ferromagnetic superconductor $\mathrm{UGe}_{2}$ has been attracting a great interest as the first material where both the superconducting (SC) state and the ferromagnetic (FM) state are sustained by $5 f$ electrons. ${ }^{1)}$ Contrary to the first expectation, it soon turned out that the mechanism of the superconductivity has nothing to do with the ferromagnetic spin fluctuations associated with FM transition. Indeed, the phase diagram of $\mathrm{UGe}_{2}$ presented in Fig. 1 , where $T_{\mathrm{C}}$ and $T_{\mathrm{SC}}$ denote the Curie temperature and the SC transition temperature, respectively, shows that the SC phase is located deep in the FM phase and a steep increase of $T_{\mathrm{C}}$ around $P=16.5$ kbar suggests the first order transition for the ferromagnetic transition. ${ }^{2)}$ This is in marked contrast with the case of the usual unconventional SC phases which appear in close proximity to and around the magnetic phase boundary since the superconductivity is mediated by the enhanced magnetic fluctuations around the second-order phase transition. The upper critical field $\left(H_{\mathrm{c} 2}(T)\right)$ at $T \rightarrow 0$ far exceeds the so-called Clogston limit for singlet pairing, $H_{\mathrm{p}}(0) \simeq 1.84 T_{\mathrm{SC}}(\mathrm{Tesla} / \mathrm{K}),{ }^{3)}$ as seen in Fig. 2. So, it may be natural to confine ourselves within a triplet manifold. While phenomenological theories based on the ferromagnetism and superconductivity appeared recently, ${ }^{4,5)}$ the mechanism of superconductivity consistent with other fundamental properties has not been clarified yet.

The superconducting mechanism appears to be related

* Present address: Institute for Solid State Physics, University of Tokyo, 5-1-5, Kashiwanoha, Kashiwa, Chiba 277-8581; email: swata@issp.u-tokyo.ac.jp to another "phase boundary" denoted by $T_{\mathrm{x}}(P)$ in Fig. 1 where a slight decrease of the resistivity has been observed. ${ }^{1,2,6)}$ As pressure increases, $T_{\mathrm{x}}$ goes down similarly to $T_{\mathrm{C}}$ and merges into the vicinity of the maximum $T_{\mathrm{SC}}$ around $P=11.4 \mathrm{kbar}$. So, it is natural to consider that the fluctuations related to $T_{\mathrm{x}}$ plays an essential role to cause the superconductivity in $\mathrm{UGe}_{2}$ as in the other unconventional superconductivity. Although the phase boundary was first identified by the small anomaly of the temperature dependence of the resistivity $\rho(T)$, it has begun to be recognized quite recently and turned out by new measurement that the uniform magnetization grows extra (other than the well developed ferromagnetic moment) for $T<T_{\mathrm{x}}{ }^{2,8-10)}$ Such metamagnetic behaviors are observed even for $P>P_{\mathrm{x}}, P_{\mathrm{x}}$ being defined by $T_{\mathrm{x}}\left(P_{\mathrm{x}}\right)=0$, under the magnetic field. Indeed, $T_{\mathrm{x}}(H)$ reappears under the magnetic field and increases as a rate of about $1 \mathrm{~K} / \mathrm{T}$ near the critical pressure $P=P_{\mathrm{x}} \cdot{ }^{9,11)}$

Furthermore, remarkable anomalies which characterize the superconductivity of $\mathrm{UGe}_{2}$ have been observed in $H_{\mathrm{c} 2}$ measurement: As shown in Fig. $2, H_{\mathrm{c} 2}$ exhibits quite unusual $T$-dependence. ${ }^{2,12)}$ Namely, $H_{\mathrm{c} 2}$ at $P=11.4$ kbar for which $T_{\mathrm{SC}}$ takes maximum merely shows gradual increase, while $H_{\mathrm{c} 2}$ at $P=13.5$ kbar shows firstorder like steep increase although $T_{\mathrm{SC}}$ at $P=13.5 \mathrm{kbar}$ is smaller than that at $P=11.4 \mathrm{kbar}$. This behavior can be also interpreted as the occurrence of the suppression of $H_{\mathrm{c} 2}(T \rightarrow 0)$ at $P=11.4$ kbar by the magnetic field. Such unusual aspects can be understood if the superconductivity is induced by the fluctuations associated with the transition at $T_{\mathrm{x}}$ as will be discussed in detail in this paper.

So far, the nature of the transition at the phase bound- 


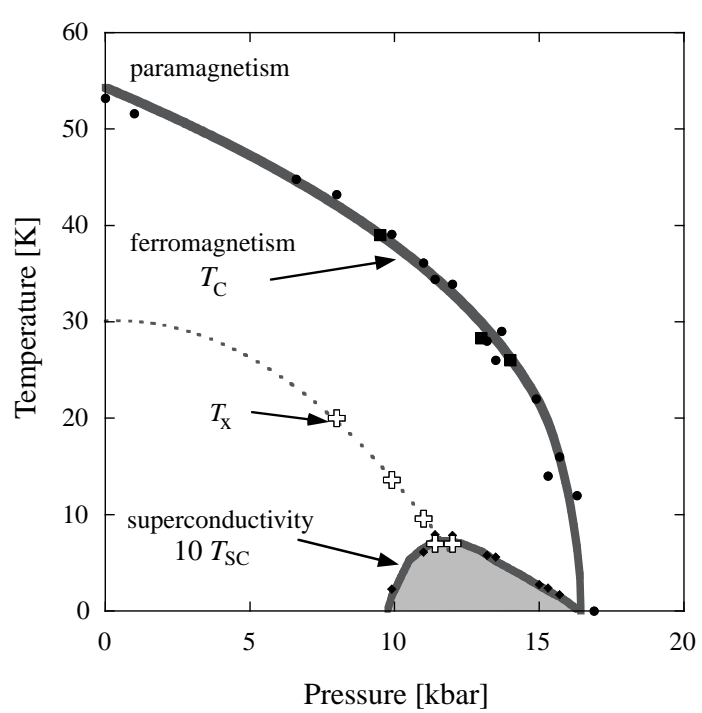

Fig. 1. Temperature-pressure phase diagram of $\mathrm{UGe}_{2} \cdot{ }^{2)} T_{\mathrm{C}}$ and $T_{\mathrm{SC}}$ denote Curie temperature and superconducting transition temperature, respectively. The cusp anomaly in the resistivity is observed at $T=T_{\mathrm{x}}$ (see text).

ary $T=T_{\mathrm{x}}(P)$ has not been established yet. However, there are some circumstantial evidences that the transition may be the coupled SDW and CDW onset due to the imperfect nesting of the majority-spin band.

The first evidence is that the Fermi surfaces of the majority-spin band obtained by the band-structure calculations is nearly nested both in the $a$ - and $c$ direction. ${ }^{13,14)}$ Although the first stage of calculation has been done on the basis of incorrect crystal symmetry due to the confusion of experiments, recent calculation by H. Yamagami, taking the correct crystal symmetry and correct easy-axis ( $a$ axis) of magnetization, also shows that the Fermi surfaces are mainly composed of $5 f$ electrons and have the structure which favors the imperfect nesting in the planes perpendicular to the $a$ - and $c$-axis. ${ }^{15)}$ So, it is expected that the nesting tendency is realized in some direction connecting the flat part of the Fermi surfaces leading to the enhanced CDW fluctuations of the majority-spin band around $T=T_{\mathrm{x}}$. It is noted that CDW fluctuations of majority-spin band is inevitably accompanied by SDW fluctuations. This picture is verified by the fact that the ferromagnetic moment of the unit cell is almost constant, $M \simeq 1.0 \mu_{\mathrm{B}}$, along $\left.T_{\mathrm{x}} \cdot 2,9,10\right)$

The second one is the existence of large lattice entropy observed by the specific heat $C$ below $T \sim T_{\mathrm{x}}<T_{\mathrm{C}}$. Integrating $C / T$ with respect to $T$ from $T=0 \mathrm{~K}$ to $T_{\mathrm{C}}$, the molar entropy is estimated to be $22 \mathrm{~J} /$ mole $\simeq 2.6 \times N_{\mathrm{A}} k_{\mathrm{B}}$ which is far larger than the entropy associated with spin degrees of freedom, $\ln 2 \times N_{\mathrm{A}} k_{\mathrm{B}}$. The molar entropy due to the Fermi quasiparticles is estimated to be $1.7 \mathrm{~J} / \mathrm{mole}$ by means of the Sommerfeld constant $\lim _{T \rightarrow 0} C / T \simeq$ $0.0314 \mathrm{~J} / \mathrm{mole} \cdot \mathrm{K}$. This value is much smaller than the observed one. The entropy due to the Debye phonon also gives only $1 / 3$ of the observed one (see the discussion in $\S 3)$. Then the extra entropy should be ascribed to the other degrees of freedom. One probable candidate is the softened optical modes coupled to the CDW instability of the majority-spin band.

The third one is the fact that the lattice structure of $\mathrm{UGe}_{2}$ is similar to that of $\alpha$-Uranium in which the CDW has been confirmed experimentally by neutron scattering in consistent with the nesting vector calculated by the band theory. ${ }^{16)} \quad \alpha$-Uranium has the zig-zag chain arrangement of $\mathrm{U}$ atoms along the $\mathrm{c}$ axis. $\mathrm{UGe}_{2}$ also has the the zig-zag chain arrangement of $U$ atoms along the a axis and Ge atoms are located between $\mathrm{U}$ atoms. Moreover, the $T$-dependences of $\rho(T)$ and $C(T)$ of $\alpha$ Uranium are similar to those of $\mathrm{UGe}_{2}$ : The cusp anomaly of $\rho(T)$ and the convex curvature of $C(T)$ at $T_{\mathrm{CDW}}$ have been also observed in $\alpha$-Uranium. Here, one may expect that $\rho(T)$ should raise if the CDW ordering occurs. Of course in the case of the perfect nesting, the gap is created at the entire region of the Fermi surface so that the system becomes insulator. However, in the case of the imperfect nesting, the situation is not so simple. The $T$-dependence of resistivity is determined by the competition between two opposite factors: One is the tendency that makes the system insulator by the gap creation at some parts of the Fermi surface. The other is the effect of the reduction of the scattering amplitude among quasi particles by the CDW ordering that makes the resistivity lower. We remark that $\mathrm{NbSe}_{2}$ where the CDW ordering due to the imperfect nesting has been confirmed experimentally also shows the similar $\rho(T)$ which decreases below $T_{\mathrm{CDW}}{ }^{17)}$

The fourth one is a rather sharp decrease of the residual resistivity $\rho(T \rightarrow 0)$ across $P=P_{\mathrm{x}}$ when $P$ is increased, ${ }^{2,18)}$ implying that the area of the Fermi surface recovers as the coupled CDW and SDW ordering vanishes at $P=P_{\mathrm{x}}$. This is because the $\rho(T \rightarrow 0)$ depend directly on the carrier number or the area of the Fermi surface and the scattering mechanism is essentially unchanged across $P=P_{\mathrm{x}}$, in contrast to the case where $T$ changes across $T_{\mathrm{x}}$ with the pressure $P$ fixed.

In this paper, we show that fundamental properties of $\mathrm{UGe}_{2}$ mentioned above can be understood in a unified picture that the superconductivity is mediated by coupled CDW and SDW fluctuations due to imperfect nesting in the majority-spin band at $T=T_{\mathrm{x}}$. In $\S 2$, we show that the growth of extra magnetization for $T<T_{\mathrm{x}}$ is understood as a mode-coupling effect of the coupled CDW-SDW ordering. In $\S 3$, we show that the anomalously large entropy around $T=T_{\mathrm{x}}(P=0)$ can be explained as contributions from optical phonons softened by the Kohn effect due to the onset of CDW ordering of the majority-spin band. In $\S 4$, we show that the pairing interaction among quasiparticles in the majority-spin band is induced by the ferromagnetic fluctuations associated with coupled CDW and SDW fluctuations enhanced around $P=P_{\mathrm{x}}$. Namely, the spin susceptibility $\chi_{\mathrm{s}}\left(q \sim 0, \mathrm{i} \omega_{m}\right)$ is enhanced by the mode-coupling effect of CDW and SDW fluctuations just as these give rise to the excess uniform magnetization below $T<T_{\mathrm{x}}$. These fluctuations induce the spin-triplet "p"-wave paring. It is shown that the anomalous temperature dependence of the upper critical field $H_{\mathrm{c} 2}(T)$ observed at $P=11.4 \mathrm{kbar}$ 




Fig. 2. Upper critical field for the field applied in parallel to the easy axis ( $a$-axis) at various pressures in $\mathrm{UGe}_{2} \cdot{ }^{2,12)}$

and $P=13.5$ kbar can be understood by the strongcoupling formalism on the basis of the present mechanism and the experimental fact that $T_{\mathrm{x}}$ is an increasing function of $H^{12}$ ) Throughout this paper we use the unit such that the Boltzmann constant $k_{\mathrm{B}}=1$ and the Plank constant $\hbar=1$ unless they are explicitly written down.

\section{§2. Mode-coupling between CDW and SDW}

In this section, we show that the excess growth of uniform magnetization can occur below $T<T_{\mathrm{x}}$ if the transition at $T=T_{\mathrm{x}}$ is the coupled CDW-SDW ordering. Such a growth of the magnetization in $\mathrm{UGe}_{2}$ has been observed by elastic neutron scattering, ${ }^{2)}$ and direct measurements of magnetization by ac-method. ${ }^{2,9,10)}$ In other words, it turns out that a central assumption of the present scenario is supported by a direct experimental evidence.

An ingredient of such a growth of magnetization below $T<T_{\mathrm{x}}$ is the fact that in the free energy there appears a mode-coupling term among the uniform magnetization, $M_{0}$, the order parameter of CDW, $N_{\mathbf{Q}}$, and of SDW, $M_{-\mathbf{Q}}$, with a nesting vector $\mathbf{Q}$, in the region $T<T_{\mathrm{X}}$ :

$$
F=F_{0}+\left[C\left(\mathbf{Q}, i \omega_{n}=0\right) M_{0} N_{\mathbf{Q}} M_{-\mathbf{Q}}+\text { c.c. }\right],
$$

where $F_{0}$ denotes the free energy other than the modecoupling term. Here we note that the mode-coupling term with uniform charge $N_{0}, M_{\mathbf{Q}}$ and $M_{-\mathbf{Q}}$ can also appear in the free-energy expansion. However, this term is considered to be suppressed by the long-range Coulomb interaction which works to maintain the charge neutrality. The mode-coupling coefficient $C\left(\mathbf{Q}, i \omega_{n}=0\right)$ in eq. (1) can be calculated in terms of the Feynman diagram shown in Fig. 3(a):

$$
\begin{aligned}
& C\left(\mathbf{Q}, i \omega_{n}=0\right)=g_{0}^{\mathrm{s}} g_{\mathbf{Q}}^{\mathrm{c}} g_{-\mathbf{Q}}^{\mathrm{s}} \\
& \times \int \frac{d^{3} p}{(2 \pi)^{3}} \frac{1}{4 T} \cosh ^{-2}\left(\frac{\varepsilon(\mathbf{p})-\mu}{2 T}\right) \frac{1}{\varepsilon(\mathbf{p})-\varepsilon(\mathbf{p}+\mathbf{Q})},(2)
\end{aligned}
$$

where $\varepsilon(\mathbf{p})$ is the dispersion of the majority-spin band and $\mu$ is the chemical potential. The coefficients in eq. $(2), g_{0}^{\mathbf{s}}(>0), g_{\mathbf{Q}}^{\mathrm{c}}(>0)$, and $g_{-\mathbf{Q}}^{\mathbf{s}}(>0)$, denote the coupling between quasiparticles and spin fluctuations with $q=0$, charge fluctuations with $\mathbf{q}=\mathbf{Q}$, and spin fluctuations with $\mathbf{q}=-\mathbf{Q}$, respectively.

First, we consider the perfect-nesting case. The perfect nesting occurs in the bipartite lattices at half filling with $\mu=0$. In this case, the relation $\varepsilon(\mathbf{p}+\mathbf{Q})=-\varepsilon(\mathbf{p})$ holds and the mode-coupling coefficient is given as follows:

$$
\begin{aligned}
C\left(\mathbf{Q}, i \omega_{n}=0\right) & =g_{0}^{\mathrm{s}} g_{\mathbf{Q}}^{\mathrm{c}} g_{-\mathbf{Q}}^{\mathrm{s}} \Omega \int d \xi D(\xi) \frac{1}{2 \xi} \frac{1}{4 T} \cosh ^{-2}\left(\frac{\xi}{2 T}\right) \\
& \left.\simeq g_{0}^{\mathrm{s}} g_{\mathbf{Q}}^{\mathrm{c}} g_{-\mathbf{Q}}^{\mathrm{s}} \Omega \frac{d D(\xi)}{d \xi}\right|_{\xi=0}
\end{aligned}
$$

where $D(\xi) \Omega=\int d^{3} p /(2 \pi)^{3} \delta(\varepsilon(\mathbf{p})-\mu-\xi)$ is the density of states measured from at the Fermi level, $\mu$, with $\Omega$ being the volume of the system. The last approximate equality in eq. (3) is valid when $T_{\mathrm{x}}$ is assumed to be much smaller than the bandwidth of quasiparticles. Therefore, the coefficient $C$ vanishes as far as the $D(\xi)$ is symmetric function of $\xi$ at $\xi \sim 0$. This result can be understood as follows: As is well known, the number density of the majority spin band, under the fixed chemical potential, remains unchanged even under the growth of the coupled CDW and SDW ordering, $\Delta_{\mathrm{C}-\mathrm{S}} \propto M_{0} N_{\mathbf{Q}} M_{-\mathbf{Q}}$, in such a case. This can easily be seen if one considers the onedimensional simple lattice as an example in Fig. 3(b): By the CDW-SDW transition the rearrangement of the charges with majority spins occurs but the net magnetization does not change as shown in Fig. 3(c).

In case that $D(\xi)$ has a weak asymmetric part with respect to $\xi=0$, the coefficient $C$ does not vanish so that the number density of majority spin band changes in general. However, it may be reasonable to assume $D(\xi)$ is symmetric around $\xi=0$ in the case where the perfect nesting is realized as in two-dimensional(2D) and three dimensional(3D) Hubbard model with only nearest neighbor hopping. In one-dimensional case, however, $D(\xi)$ has a weak asymmetry in general under the condition of perfect nesting. In such a case, the sign of the magnetization change cannot be determined by the sign of the term of $\mathcal{O}\left(\Delta_{\mathrm{C}-\mathrm{S}}^{2}\right)$, but is determined by the sign of the term with logarithmic correction, $\Delta_{\mathrm{C}-\mathrm{S}}^{2} \ln \Delta_{\mathrm{C}-\mathrm{S}}$. In any case, the effect is small one proportional to $|d D(\xi) / d \xi|_{\xi=0} \mid \ll D(\xi=0)$, in contrast to the case of inperfect nesting where the effect is proportional to $D(\xi=0)$ as will be discussed below.

Next we consider the imperfect-nesting case. In this case, the energy gap opens at the Fermi surface on the zone boundary transferred by the nesting vector $\mathbf{Q}$. Hence, the coefficient eq. (2) does not diverge but has a finite value. The important point is that the sign of $C\left(\mathbf{Q}, i \omega_{n}=0\right)$ changes according to the relative positions between the Fermi surface and the nesting zone boundary.

As an example let us consider the majority-spin band on the two-dimensional square lattice

$$
\varepsilon(\mathbf{p})=-2 t\left\{\cos \left(p_{x}\right)+\cos \left(p_{y}\right)\right\}-4 t^{\prime} \cos \left(p_{x}\right) \cos \left(p_{y}\right),
$$


with the nearest-neighbor hopping $t$ and the nextnearest-neighbor hopping $t^{\prime}$. In Fig. 3(d) and Fig. 3(e) the Fermi surface with $\mu=0$ and nesting zone boundary is plotted by the solid line and the dashed line, respectively.

For positive $t^{\prime}$ the Fermi surface with $\mu=0$ is located inside the nesting zone boundary as in Fig. 3(d). In this case the mode-coupling coefficient has a negative value $C\left(\mathbf{Q}, i \omega_{n}=0\right)<0$. Namely, in this case the uniform magnetization $M_{0}$ increases for $T<T_{\mathrm{x}}$. This can be understood as follows: The energy gap opens at the part of the Fermi surface along the nesting zone boundary, which is drawn by the shaded spots in Fig. 3(d). By the gap creation, the energy band inside the zone boundary shifts downward. As for the area at which the gap opens the situation is the same as the perfect nesting case. Namely, the shaded spots in Fig. 3(d) do not contribute to the change of uniform magnetization. However, the important difference from the perfect-nesting case is that the Fermi surface which deviates from the nesting zone boundary (i.e., the part where the gap does not open) shifts downward by the gap creation. Hence, the electrons flow into the majority-spin band from the minority-spin band so that the uniform magnetization increases.

On the contrary, for negative $t^{\prime}$ the Fermi surface with $\mu=0$ is located outside the nesting zone boundary as in Fig. 3(e). In this case the energy band shifts upward by the gap creation and it is shown that the mode-coupling coefficient has a positive value $C\left(\mathbf{Q}, i \omega_{n}=0\right)>0$. Namely, in this case electrons flow into the minority-spin band from the majority-spin band so that the uniform magnetization $M_{0}$ decreases for $T<T_{\mathrm{x}}$.

Here we have discussed the change of the uniform magnetization referring to the one- and two-dimensional lattices as typical examples to draw a general conclusion. In the real systems, the shape of the Fermi surface is often more complicated, but our statement derived here can be applied to the Fermi surface transferred by the nesting vector $\mathbf{Q}$. As for $\mathrm{UGe}_{2}$, the Fermi surface of the majority-spin band is located inside the nesting zone boundary according to the recent band-structure calculation. ${ }^{13)}$ Namely, it seems to correspond to the case (d) in above examples, which should exhibit the increase of the uniform magnetization for $T<T_{\mathrm{x}}$ as observed experimentally.$^{2,9,10)}$

Concluding this chapter, let us remark that the mechanism of magnetization changes due to simultaneous ordering of CDW and SDW with the equal nesting wave vector is quite general one which may be found in other systems with such a property. It is also remarked that the sign of magnetization change depends on the curvature of the Fermi surface near the nesting vector as discussed above.

\section{§3. Specific heat due to Kohn anomaly}

In this section, we discuss how the temperature dependence of the specific heat $C(T)$ at $T<T_{\mathrm{C}}$, especially a gentle hump around $T=T_{\mathrm{x}}$, is understood from the present point of view that $T_{\mathrm{x}}$ is the onset temperature (a)

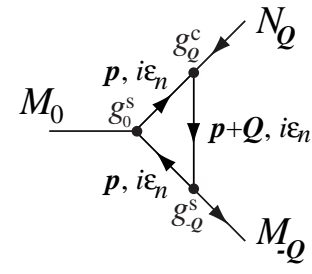

(b)

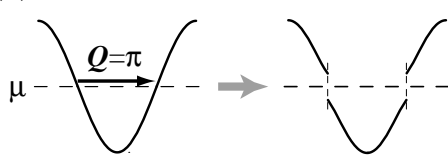

(d)

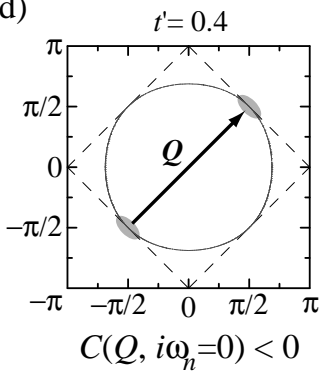

(c)

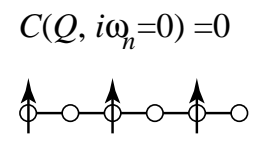

(e)

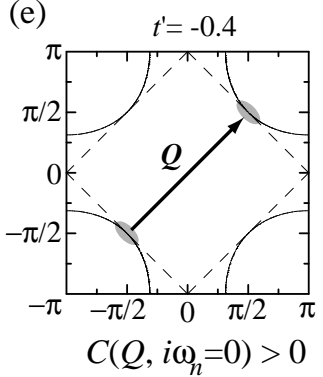

Fig. 3. (a) The mode-coupling term between the CDW and the SDW in the lowest order. The band picture (b) and the realspace picture (c) by the perfect nesting in the majority-spin band in the one-dimensional simple lattice. The Fermi surface with $\mu=0$ (solid line) and the nesting zone boundary (dashed line) in the two-dimensional square lattice in the majority-spin band $\varepsilon(\mathbf{p})=-2\left\{\cos \left(p_{x}\right)+\cos \left(p_{y}\right)\right\}-4 t^{\prime} \cos \left(p_{x}\right) \cos \left(p_{y}\right)$ with (d) $t^{\prime}=$ 0.4 and $(\mathrm{e}) t^{\prime}=-0.4$. The imperfect nesting by vector $\mathbf{Q}=$ $(\pi, \pi)$ causes the gap at the shaded spots.

of coupled CDW-SDW ordering. The specific heat due to the Debye phonon cannot afford to explain the global behavior of $C(T) / T$ as can be seen in Fig. 4 in which the Debye contribution $C_{\mathrm{D}} / T$ is compared with the experiment at ambient pressure. ${ }^{2)}$ The Debye contribution is given as

$$
\frac{C_{\mathrm{D}}}{T}=\Lambda\left[\frac{4}{T^{2}} \int_{0}^{\omega_{\mathrm{D}}} \mathrm{d} \omega \frac{\omega^{3}}{e^{\omega / T}-1}-\frac{\omega_{\mathrm{D}}^{4}}{T^{2}} \frac{1}{e^{\omega_{\mathrm{D}} / T}-1}\right],
$$

with the coefficient $\Lambda$ defined by

$$
\Lambda \equiv \frac{9}{2} \frac{N k_{\mathrm{B}}}{\omega_{\mathrm{D}}^{3}},
$$

where $N$ and $\omega_{\mathrm{D}}$ denote the unit-cell number included in the system and the averaged Debye energy, respectively. In the low temperature limit, $T \ll \omega_{\mathrm{D}}$, eq. (4) is reduced to the conventional form as

$$
\frac{C_{\mathrm{D}}}{T} \simeq N k_{\mathrm{B}} \frac{12 \pi^{4}}{5 \omega_{\mathrm{D}}^{3}} T^{2}
$$

Comparing the experimental value for the coefficient of $T^{2}$ term of eq. (6), $3.2 \times 10^{-4} \mathrm{~J} / \mathrm{K}^{4} \cdot$ mole, ${ }^{2)}$ we obtain the Debye energy as $\omega_{\mathrm{D}}=182 \mathrm{~K}$. Using this value for $\omega_{\mathrm{D}}$, we have ploted $C_{\mathrm{D}} / T$ in Fig. 4 . In order to account for the hump around $T_{\mathrm{x}}$, there should be an extra contribution to the entropy from other than the magnetic origin because the difference between $C_{\mathrm{D}} / T$ and the experimental 
value is far larger than the jump of $C / T$ at $T=T_{\mathrm{C}}$, the ferromagnetic transition.

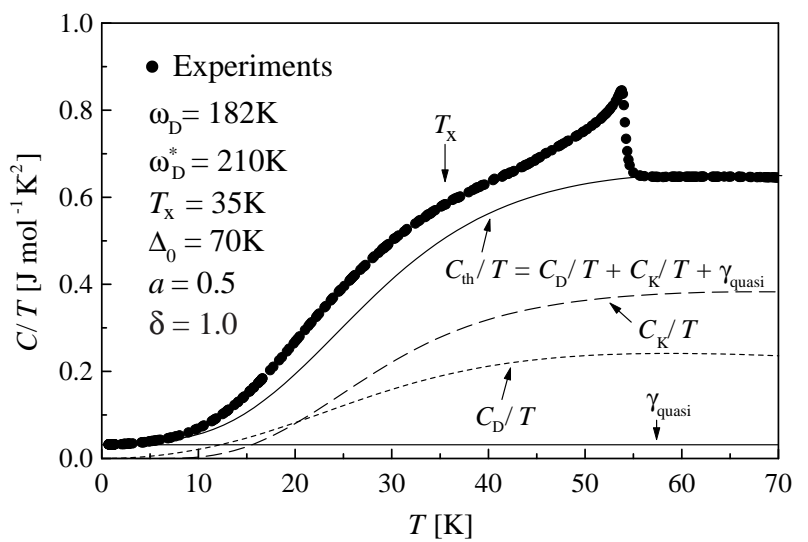

Fig. 4. $C / T$ vs $T$. The solid circles represent the experimental values, ${ }^{2)}$ the solid line the theoretical ones, which consist of the Debye contribution (dotted line), the cotributions from optical modes with the Kohn anomaly (dashed line), and the fermionic contributions from quasiparticles (solid line).

The optical phonons are subject to the Kohn anomaly if the Fermi surface of quasipartices is nested as expected in the majority-spin band of $\mathrm{UGe}_{2}$. We parameterize the dispersion of optical phonons exhibiting the Kohn anomaly as follows:

$$
\varepsilon(\mathbf{k})=\sqrt{\left(s_{\|} k_{z}\right)^{2}+s_{\perp}^{2}\left(k_{x}^{2}+k_{y}^{2}\right)^{2}+\Delta^{2}},
$$

where the wave vector $\mathbf{k}$ is measured from the nesting vector $\mathbf{Q}$, and we here take the direction along which the nesting occurs as $k_{z}$-direction without loss of generality. The gap $\Delta$ parameterizes the degree of softening of the phonons, and $s_{\|}$and $s_{\perp}$ denote the sound velocities for longitudinal and transverse directions, respectively, with respect to $\mathbf{Q}$. There exist two independent optical-phonon modes corresponding to two Ge ions in the unit cell. For simplicity, we assume that the dispersions of two modes are identical. Then, the energy due to optical-phonon modes with Kohn anomaly is given as

$$
E_{\mathrm{K}}=\Xi \int_{0}^{\omega_{\mathrm{D}}^{*}} \mathrm{~d} \omega_{z} \int_{0}^{\omega_{\mathrm{D}}^{*}} \mathrm{~d} \omega \omega \frac{\varepsilon\left(\omega_{z}, \omega\right)}{e^{\varepsilon\left(\omega_{z}, \omega\right) / T}-1},
$$

where $\omega_{z} \equiv s_{\|} k_{z}, \omega \equiv s_{\perp} \sqrt{k_{x}^{2}+k_{y}^{2}}, \delta \equiv s_{\perp} / s_{\|}$. The Debye energy $\omega_{\mathrm{D}}^{*}$ is different from $\omega_{\mathrm{D}}$ for the acoustic modes in general.

In the high temperature limit, $T \gg \omega_{\mathrm{D}}^{*}$, eq. (8) is reduced to

$$
E_{\mathrm{K}}=\Xi \int_{0}^{\omega_{\mathrm{D}}^{*}} \mathrm{~d} \omega_{z} \int_{0}^{\omega_{\mathrm{D}}^{*}} \mathrm{~d} \omega \omega T=\frac{1}{2} \Xi T \omega_{\mathrm{D}}^{* 3} .
$$

The coefficient $\Xi$ is determined so as to reproduce the value of Dulong-Petit:

$$
\Xi=\frac{6 N k_{\mathrm{B}}}{\omega_{\mathrm{D}}^{* 3}} \times 2
$$

The specific heat $C_{\mathrm{K}}$ is obtained from eq. (8) as follows:

$$
\begin{array}{r}
\frac{C_{\mathrm{K}}}{T}=\Xi\left[\frac{1}{T^{3}} \int_{0}^{\omega_{\mathrm{D}}^{*}} \mathrm{~d} \omega_{z} \int_{0}^{\omega_{\mathrm{D}}^{*}} \mathrm{~d} \omega \omega \frac{\varepsilon^{2} e^{\varepsilon / T}}{\left(e^{\varepsilon / T}-1\right)^{2}}\right. \\
\left.-\frac{1}{2 T} \frac{\partial \Delta^{2}}{\partial T} \int_{0}^{\omega_{\mathrm{D}}^{*}} \mathrm{~d} \omega_{z} \int_{0}^{\omega_{\mathrm{D}}^{*}} \mathrm{~d} \omega \frac{\omega}{\varepsilon} \frac{(\varepsilon / T-1) e^{\varepsilon / T}+1}{\left(e^{\varepsilon / T}-1\right)^{2}}\right] .
\end{array}
$$

The second term crucially depends on the temperature dependence of the gap $\Delta$ defined as the lowest opticalphonon energy in eq. (7). To discuss the relationship between the temperature dependence of the specific heat and the effect of the electron-phonon coupling, we show the Dyson equation for the phonon Green's function $D\left(\mathbf{q}, \mathrm{i} \omega_{n}\right)$ in Fig. 5(a). Here $D_{0}\left(\mathbf{q}, \mathrm{i} \omega_{n}\right)$ denotes the bare Green's function for phonons. $\Pi$ and $\Sigma_{\mathrm{MC}}$ denote the electron polarization function and the selfenergy for the mode coupling, respectively. The softening of the optical-phonon mode with gap $\Delta$ at the wave vector $\mathbf{Q}$ which should be the same as the nesting vector is illustrated in Fig. 5(b). On the mean-field level where $\Sigma_{\mathrm{MC}}$ is set to be zero, the $T$-dependence of $\Delta$ is given near the ordering temperature $T_{\mathrm{x}}^{\mathrm{MF}}$ like

$$
\Delta_{\mathrm{MF}}^{2} \simeq J\left|T-T_{\mathrm{x}}^{\mathrm{MF}}\right|,
$$

where $J$ is a positive constant which is determined by the band structure of quasiparticles and the electronphonon coupling constant. Then, $C_{\mathrm{K}} / T$ given by eq. (11) exhibits a discontinuous jump at $T=T_{\mathrm{x}}^{\mathrm{MF}}$ through the factor $\partial \Delta^{2} / \partial T$ as shown in Fig. 5(c). However, in case the mode-coupling term cannot be neglected, in the limit $\Delta \rightarrow 0$ the mode-coupling effect gives the self-energy of the relevant optical phonon mode the term like $-M_{1}+$ $M_{2} \Delta, M_{1}$ and $M_{2}$ being positive constants with weak temperature dependence near $T_{\mathrm{x}}$ as discussed in ref. ${ }^{19)}$ (see Fig. 5(d)). Thus the self-consistent equation for $\Delta$ takes the following form at $T>T_{\mathrm{x}}, T_{\mathrm{x}}$ being the ordering temperature determined self-consistently as below:

$$
\Delta^{2} \simeq J\left(T-T_{\mathrm{x}}^{\mathrm{MF}}\right)+M_{1}-M_{2} \Delta .
$$

By solving this equation near $T_{\mathrm{x}} \equiv T_{\mathrm{x}}^{\mathrm{MF}}-\left(M_{1} / J\right)$, we obtain

$$
\Delta^{2}=J^{2}\left(T-T_{\mathrm{x}}\right)^{2} / M_{2}^{2} .
$$

For $T<T_{\mathrm{x}}$, similar temperature dependence is obtained by solving much more complicated equation due to the existence of ordering. Namely, it turns out that in case the effect of the mode coupling is relevant, the jump in the specific heat at the transition temperature $T_{\mathrm{x}}$ is smeared out by the fluctuations. We consider that $\mathrm{UGe}_{2}$ may correspond to such a case where the mode-coupling term cannot be neglected for the fluctuations due to the strong electron-phonon coupling. Thus, to calculate the specific heat of $\mathrm{UGe}_{2}$ we introduce a parameterization for $T$-dependence of $\Delta$ as follows:

$$
\Delta=\frac{\Delta_{0}^{2}\left(T^{2}-T_{\mathrm{x}}^{2}\right)^{2}}{T_{\mathrm{x}}^{4}+a T^{4}} .
$$

This is reduced in low- and high-temperature limits as 
follows:

$$
\Delta^{2}(T) \simeq \begin{cases}\Delta_{0}^{2}, & T \ll T_{\mathrm{x}} \\ \frac{\Delta_{0}^{2}}{a}, & T \gg T_{\mathrm{x}}\end{cases}
$$

(a)

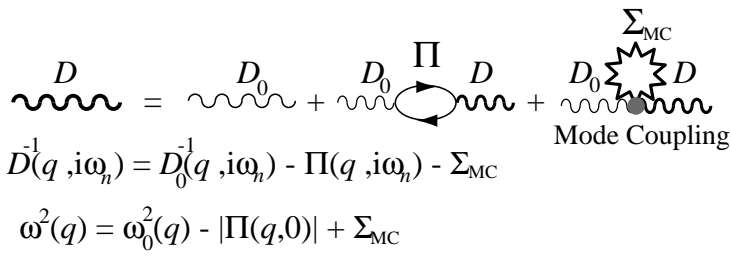

(b)

(c)
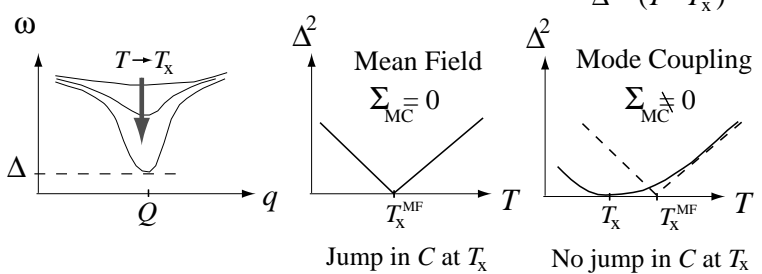

Fig. 5. (a) Dyson equation of phonon Green's function including the selfenergy of the mode coupling. (b) Softening of optical phonon mode at the nesting vector $\mathbf{Q}$. Temperature dependence of the gap in the mean-field framework (c) and in the framework including the mode-coupling term $(\mathrm{d})$.

In Fig. 4, the contribution from eq. (11) is shown as dashed line for parameters $\omega_{\mathrm{D}}^{*}=210 \mathrm{~K}, \Delta_{0}=70 \mathrm{~K}, a=0.5$, and $\delta=1.0$. One solid line in Fig. 4 is a contribution from the Fermi quasiparticles $\gamma_{\text {quasi }}$ which is determined experimentally as $\gamma_{\text {quasi }}=\lim _{T \rightarrow 0} C / T \simeq 0.0314 \mathrm{~J} / \mathrm{mole}$ $\mathrm{K}^{2}{ }^{2)}$ The other solid line is for $C_{\mathrm{th}} / T$ which is the sum of $C_{\mathrm{D}} / T, C_{\mathrm{K}} / T$, and $\gamma_{\text {quasi }}$. The curve $C_{\mathrm{th}} / T$ reproduces quite well the global behavior of observed $C / T$. In Fig. 6, $C / T$ (solid circle), $C_{\text {th }} /$ (solid line), and their difference $\Delta C / T \equiv C / T-C_{\mathrm{th}} / T$ (solid square) are shown. The origin of $\Delta C / T$ near $T=T_{\mathrm{C}}$ is apparently the magnetic entropy of local component of magnetization, while that of a hump around $T=30 \mathrm{~K}$ may be attributed to the mass enhancement due to the criticality of coupled CDW-SDW ordering at $T=T_{\mathrm{x}} \simeq 35 \mathrm{~K}$, where the enhancement of a few times of $\gamma_{\text {quasi }}$ is expected as observed at $\left.(T, P) \simeq\left(0, P_{\mathrm{x}}\right)^{6}{ }^{6}\right)$

If we had not taken into account the effect of Kohn anomaly, we could not account for the gentle hump of $C / T$ around $T=35 \mathrm{~K}$ by the Einstein model which simulates the two optical phonons with frequency $\omega_{\mathrm{E}}$. Indeed, its contribution $C_{\mathrm{E}} / T$ is given as

$$
\frac{C_{\mathrm{E}}}{T}=\frac{6 N k_{\mathrm{B}}}{T^{3}} \frac{\omega_{\mathrm{E}} e^{\omega_{\mathrm{E}} / T}}{\left(e^{\omega_{\mathrm{E}} / T}-1\right)^{2}} .
$$

This has a rather sharp maximum $C_{\mathrm{E}}^{\max } / T \simeq 76 / \omega_{\mathrm{E}}^{2} \mathrm{~J}$ $\mathrm{mol}^{-1} \mathrm{~K}^{-2}$ at $T \simeq 0.39 \omega_{\mathrm{E}}$; so that it is difficult to reproduce a broad hump structure of $C / T$ observed by experiments.

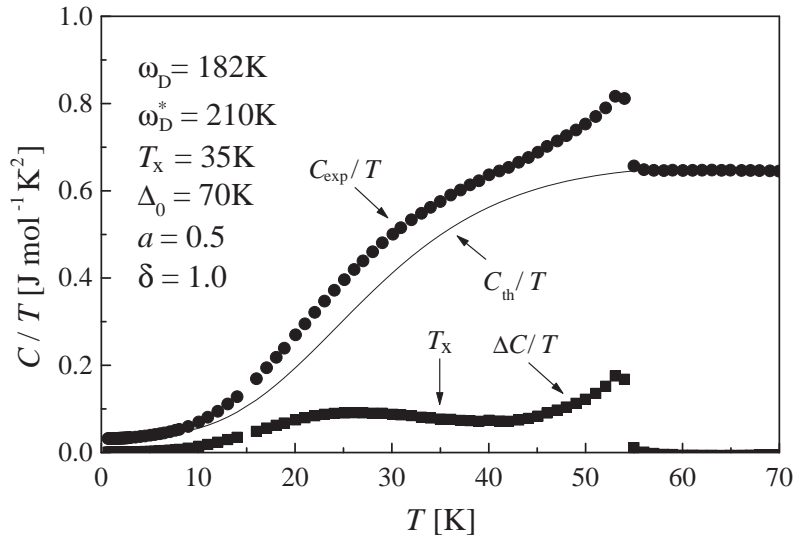

Fig. 6. Difference of $C / T$ between experiment and model. The solid circles represent the experimental values, ${ }^{2)}$ solid line theoretical ones of model, and the solid squares the differences.

\section{§4. Pairing Interaction and Strong-Coupling Ef- fect on $H_{\mathrm{c} 2}$}

As we discussed in $\S 2$, the excess ferromagnetic component begins to arise at $T=T_{\mathrm{x}}$, where the uniform spin susceptibility is diverging due to the mode-coupling effect among charge and spin fluctuations with the nesting vector $\mathbf{Q}$. While the charge susceptibility can be also diverging at $T=T_{\mathrm{x}}$ due to the mode-coupling effect among the uniform charge $N_{\mathbf{q}=0}$ and two modes of SDW, $M_{\mathbf{Q}}$ and $M_{-\mathbf{Q}}$, uniform charge fluctuations are suppressed by the long-range Coulomb interaction which works to maintain the charge neutrality as mentioned in $\S 2$. Then, the pairing interaction between quasiparticles with majority spin, due to those mode-coupling effects, is mediated mainly by the coupled two modes of the spin susceptibility $\chi_{\mathrm{s}}$ and the charge susceptibility $\chi_{\mathrm{c}}$ as shown in Fig. $7(\mathrm{a})$, but not by the coupled modes of $\chi_{\mathrm{s}}$ and $\chi_{\mathrm{s}}$. The coupled fluctuations of $\chi_{\mathrm{s}}$ and $\chi_{\mathrm{c}}$ which are related to the excess ferromagnetic moment are regarded as the ferromagnetic spin fluctuation $\tilde{\chi}_{\mathrm{s}}$ which is enhanced for the small momentum transfer, i.e., $\mathbf{q} \sim 0$. The important point is that the coupled two modes can make the scattering wave vectors for the Cooper channel, $\mathbf{q}=\mathbf{k}-\mathbf{k}^{\prime}$, zero which is profitable for the triplet pairing (see Fig. 7(a)), in marked contrast to the singlemode case where $\chi_{\mathrm{s}}$ or $\chi_{\mathrm{c}}$ with finite $\mathbf{Q}$ gives a small repulsive part for the triplet pairing as has been well recognized. ${ }^{20-23)}$

These coupled fluctuation propagators give non-zero contributions even for the external wave vector $q=0$ and the bosonic matsubara frequency $\omega_{m} \neq 0, \omega_{m} \equiv 2 m \pi T$. If we parameterize these propagators as

$$
\begin{aligned}
\chi_{\mathrm{s}}\left(\mathbf{Q}+\mathbf{q}, \mathrm{i} \omega_{m}\right) & \simeq \chi_{\mathrm{c}}\left(\mathbf{Q}+\mathbf{q}, \mathrm{i} \omega_{m}\right) \\
& =\frac{\chi_{0}}{\eta_{0}+A_{0} q^{2}+C_{0}\left|\omega_{m}\right|},
\end{aligned}
$$

the propagator $\tilde{\chi}_{\mathrm{s}}$ of the coupled fluctuations is given as

$$
\tilde{\chi}_{\mathrm{s}}\left(q, \mathrm{i} \omega_{m}\right)=T \sum_{\omega_{m^{\prime}}} \sum_{\mathbf{q}^{\prime}} \chi_{\mathrm{s}}\left(\mathbf{Q}+\mathbf{q}^{\prime}, \omega_{m^{\prime}}+\omega_{m}\right)
$$




$$
\begin{aligned}
& \times \chi_{\mathrm{s}}\left(\mathbf{Q}+\mathbf{q}^{\prime}, \omega_{m^{\prime}}\right) \\
& \simeq \chi_{0}^{2} \sum_{\mathbf{q}^{\prime}} \int_{0}^{\infty} \frac{d x}{\pi} \operatorname{coth} \frac{x}{2 T} \\
& \times \frac{x}{\varphi_{q^{\prime}}^{2}+x^{2}} \frac{\left(\varphi_{\mathbf{q}+\mathbf{q}^{\prime}}+C_{0}\left|\omega_{m}\right|\right)}{\left(\varphi_{\mathbf{q}+\mathbf{q}^{\prime}}+C_{0}\left|\omega_{m}\right|\right)^{2}+x^{2}},
\end{aligned}
$$

where $\varphi_{q} \equiv \eta_{0}+A_{0} q^{2}$. It is easy to verify that $\lim _{q \rightarrow 0} \tilde{\chi}_{\mathrm{s}}\left(q, \mathrm{i} \omega_{m}\right)$ is finite (non-zero) and continuous as a function of $\omega_{m}$ and diverges in proportion to $T \chi_{0}^{2}\left(4 \pi A_{0}\right)^{-1} / \eta_{0}$ in the $2 \mathrm{D}$ system, and $T \chi_{0}^{2}\left(8 \pi A_{0}^{3 / 2}\right)^{-1} / \sqrt{\eta_{0}}$ in the $3 \mathrm{D}$ system, as approaching the onset of coupled CDW-SDW ordering at finite temperature, i.e., $\omega_{m}=0$ and $\eta_{0} \rightarrow 0$. Near the quantum critical point at $T=0$, these divergent behaviors are given as $\ln \left(1 / \eta_{0}\right)$ in the $2 \mathrm{D}$ system, and (const. $\left.-\sqrt{\eta_{0}}\right)$ in the $3 \mathrm{D}$ system, respectively. It is noted that the latter expression in the $3 \mathrm{D}$ system exhibits a sharp peak with cusp at $\eta_{0}$, while it does not diverge there.

By careful inspection of the expression of right hand side of eq. (19), one can see that the main dependence of $\tilde{\chi}_{\mathrm{s}}\left(q, \mathrm{i} \omega_{m}\right)$ on $q$ and $\omega_{m}$ arises through a combination $A_{0} q^{2}+C_{0}\left|\omega_{m}\right|$. An explicit dependence of $\tilde{\chi}_{\mathrm{s}}\left(q, \mathrm{i} \omega_{m}\right)$ on $A_{0} q^{2}+C_{0}\left|\omega_{m}\right|$ is not simple compared to those for $\chi_{\mathrm{s}}$ and $\chi_{\mathrm{c}}$, eq. (18), and depends on the space dimensionality. Indeed, the main part of $\tilde{\chi}_{\mathrm{s}}\left(q, \mathrm{i} \omega_{m}\right)$ is proportional to $T \chi_{0}^{2}\left(2 \pi A_{0}\right)^{-1}\left(A_{0} q^{2}+\right.$ $\left.C_{0}\left|\omega_{m}\right|\right)^{-1} \ln \left[\left(2 \eta_{0}+A_{0} q^{2}+C_{0}\left|\omega_{m}\right|\right) / 2 \eta_{0}\right]$ in the $2 \mathrm{D}$ system, and $T \chi_{0}^{2}\left(4 \pi A_{0}^{3 / 2}\right)^{-1} /\left[\sqrt{\eta_{0}}+\sqrt{\eta_{0}+\left(A_{0} q^{2}+C_{0}\left|\omega_{m}\right|\right) / 2}\right.$ in the $3 \mathrm{D}$ system. The corresponding expressions at $T=0$ near the quantum critical point are too complicated to be written here.

Since the purpose of the present paper is to give a theoretical basis for qualitative but unified understanding of the superconducting mechanism of $\mathrm{UGe}_{2}$, especially an origin of anomalous temperature dependence of $H_{\mathrm{c} 2}(T)$, we adopt the following phenomenological form for $\tilde{\chi}_{\mathrm{s}}\left(q, \mathrm{i} \omega_{m}\right)$ as

$$
\tilde{\chi}_{\mathrm{s}}\left(\mathbf{q}, \mathrm{i} \omega_{m}\right)=\frac{\chi_{0}}{\eta+A q^{2}+C\left|\omega_{m}\right|} .
$$

This reproduces approximately a characteristic behavior of $\tilde{\chi}_{\mathrm{s}}\left(q, \mathrm{i} \omega_{m}\right)$ near the critical point of coupled CDWSDW ordering if the parameters $\eta, A$, and $C$ are constants different from $\eta_{0}, A_{0}$ and $C_{0}$ in eq. (18) and determined so as to reproduce key experimental observations.

The $q$-dependence of the vertex connecting the coupled CDW-SDW fluctuations and the quasiparticles depends on a dynamical structure of the fluctuations. If the magnetization is the conserved quantity, the vertex for $q=0$ and $\omega_{m} \neq 0$ should vanish as can be verified by a perturbational calculation of the triangle diagram shown in Fig. 7(a). However, if the magnetization is not conserved due to the strong spin-orbit interaction at U-sites as in the present case, the vertex does not vanish even for $q=0$ and $\omega_{m} \neq 0$. The mode mediating the pairing interaction shown in Fig. 7(a) is that arising from an incoherent process in the sense of the Fermi liquid theory. It is known that such a contribution vanishes in general if the relevant quantity of the fluctuations is conserved one. ${ }^{24)}$ On the other hand, if it is not conserved one, there remains an incoherent contribution which arises from high energy processes and cannot be described by the response function of quasiparticles. It is quite crucial for the behavior of incoherent processes whether the relevant quantity is conserved or not, while it is not so severe for the quasiparticle response which mainly reflects the structure of the phase space of low energy particlehole continuum of quasiparticles as far as the effect of the impurity scattering is neglected.

On the basis of the reasoning above, the pairing interaction $V\left(\mathbf{q}, \mathbf{i} \omega_{m}\right)$ for $\mathbf{q}=\mathbf{k}-\mathbf{k}^{\prime} \sim 0$ is postulated as

$$
V\left(\mathbf{q}, \mathrm{i} \omega_{m}\right) \simeq U-\left|I^{*}\right|^{2} \tilde{\chi}_{\mathrm{s}}\left(\mathbf{q}, \mathrm{i} \omega_{m}\right),
$$

where $U$ is the short-range ( $q$-independent) repulsive interaction, $I^{*}$ is the coupling between quasiparticles and the coupled CDW-SDW fluctuations. The wavenumber dependence of $I$ is assumed to be neglected around $q \sim 0$. The pairing interaction arising from the exchange of the single mode of SDW and CDW fluctuations with the nesting vector $\mathbf{Q}$ gives a small repulsive part for the triplet p-wave interaction as mentioned above. However, this contribution is not considered to play a crucial role because the wavenumber dependence of $\chi_{\mathrm{s}}(\mathbf{q})$ and $\chi_{\mathrm{c}}(\mathbf{q})$ is weak at $\mathbf{q} \sim 0$. So, we neglect its contribution for the moment.

(a)

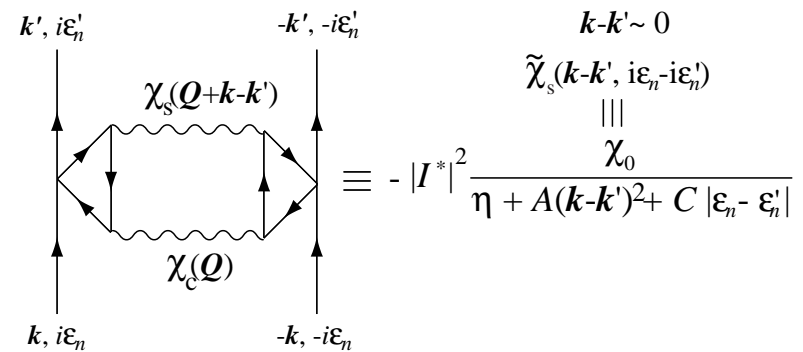

(b)

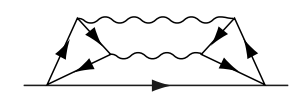

Fig. 7. (a) Paring mechanism mediated by coupled CDW $\left(\chi_{\mathrm{c}}\right)$ and SDW $\left(\chi_{\mathrm{s}}\right)$ fluctuations with the nesting vector $\mathbf{Q}$. (b) Selfenergy of electrons due to one-fluctuation exchange process.

For simplicity of calculations, we assume a threedimensional spherical band with majority spins. Then the triplet p-wave component $V(\ell=1)$ of $V\left(\mathbf{q}, \mathrm{i} \omega_{m}\right)$ for $\mathbf{q} \equiv \mathbf{k}-\mathbf{k}^{\prime}$ with $|\mathbf{k}|=\left|\mathbf{k}^{\prime}\right|=k_{\mathrm{F}}$ is given by

$$
V\left(\ell=1, \mathrm{i} \omega_{m}\right) \approx-\left|I^{*}\right|^{2} \int_{0}^{\pi} \frac{\mathrm{d} \theta}{2} \sin \theta \sqrt{3} \cos \theta \tilde{\chi}_{\mathrm{s}}\left(q, \mathrm{i} \omega_{m}\right),
$$

where $q=2 k_{\mathrm{F}}|\sin \theta / 2|$, with $\cos \theta=\left(\mathbf{k} \cdot \mathbf{k}^{\prime}\right) / k_{\mathrm{F}}^{2}$. Substituting eq. (20) for $\tilde{\chi}_{\mathrm{s}}$ and after simple algebra, we obtain

$$
V\left(\ell=1, \mathrm{i} \omega_{m}\right) \approx-\frac{\sqrt{3}\left|I^{*}\right|^{2} \chi_{0}}{2 k_{\mathrm{F}}^{2} A}
$$




$$
\times\left[-1+\frac{B+1}{2} \ln \left|\frac{(B+2)}{B}\right|\right],
$$

where $\omega$-dependent $B$ is defined as

$$
B\left(\omega_{m}\right) \equiv \frac{\eta+C\left|\omega_{m}\right|}{2 k_{\mathrm{F}}^{2} A} .
$$

The corresponding selfenergy $\Sigma$ of electrons due to the one-fluctuation exchange process (see Fig. 7(b)) is given as follows:

$$
\Sigma\left(\mathbf{p}, \mathrm{i} \epsilon_{n}\right)=T \sum_{\epsilon_{n^{\prime}}} \sum_{\mathbf{p}^{\prime}}\left|I^{*}\right|^{2} \frac{\tilde{\chi}_{\mathrm{s}}\left(\mathbf{p}-\mathbf{p}^{\prime}, \mathrm{i} \epsilon_{n}-\mathrm{i} \epsilon_{n^{\prime}}\right)}{\mathrm{i} \epsilon_{n^{\prime}}-\xi_{\mathbf{p}^{\prime}}},
$$

where $\epsilon_{n} \equiv(2 n+1) \pi T$ is the fermionic Matsubara frequency. The summation in eq. (25) with respect to $\mathbf{p}^{\prime}$ can be separated approximately and reduced to

$$
\begin{aligned}
\Sigma\left(\mathbf{p}, \mathrm{i} \epsilon_{n}\right)=T & \sum_{\epsilon_{n^{\prime}}}\left|I^{*}\right|^{2} \sum_{\mathbf{p}^{\prime}} \frac{1}{\mathrm{i} \epsilon_{n^{\prime}}-\xi_{\mathbf{p}^{\prime}}} \\
& \times \int_{0}^{\pi} \frac{\mathrm{d} \theta}{2} \sin \theta \tilde{\chi}_{\mathbf{s}}\left(q, \mathrm{i} \epsilon_{n}-\mathrm{i} \epsilon_{n^{\prime}}\right),
\end{aligned}
$$

where $q=2 k_{\mathrm{F}}|\sin \theta / 2|$. Here we have approximated as $|\mathbf{p}| \simeq\left|\mathbf{p}^{\prime}\right| \simeq k_{\mathrm{F}}$ in $\tilde{\chi}_{\mathrm{s}}$ in eq. (26). Then, the renormalized frequency $\tilde{\epsilon}_{n} \equiv \epsilon_{n}+\mathrm{i} \Sigma\left(\mathrm{i} \epsilon_{n}\right)$ is given approximately as follows:

$$
\begin{aligned}
\tilde{\epsilon}_{n}=\epsilon_{n}+\pi T \sum_{\epsilon_{n^{\prime}}} \frac{\left|I^{*}\right|{ }^{2} \chi_{0}}{2 k_{\mathrm{F}}^{2} A} \frac{N_{\mathrm{F}} \operatorname{sign}\left(\epsilon_{n^{\prime}}\right)}{2} \\
\quad \times \ln \left|\frac{B\left(\epsilon_{n}-\epsilon_{n^{\prime}}\right)+2}{B\left(\epsilon_{n}-\epsilon_{n^{\prime}}\right)}\right|,
\end{aligned}
$$

where $N_{\mathrm{F}}$ is the density of states of band electrons. In deriving eq. (27), we have discarded the real part of $\Sigma\left(\mathrm{i} \epsilon_{n}\right)$ on the understanding that it can be absorbed into the chemical-potential shift.

Here we introduce the function for renormalization effect as

$$
M\left(\omega_{m}\right)=\lambda \frac{\ln \left|\frac{\Gamma+\left|\omega_{m}\right|+2 E^{*}}{\Gamma+\left|\omega_{m}\right|}\right|}{\ln \left|\frac{\Gamma+2 E^{*}}{\Gamma}\right|},
$$

where $\lambda, \Gamma$, and $E^{*}$ are defined as

$$
\begin{gathered}
\lambda \equiv \frac{\left|I^{*}\right|^{2} \chi_{0}}{2 k_{\mathrm{F}}^{2} A} \frac{N_{\mathrm{F}}}{2} \ln \left|\frac{\Gamma+2 E^{*}}{\Gamma}\right|, \\
\Gamma \equiv \frac{\eta}{C}
\end{gathered}
$$

and

$$
E^{*} \equiv \frac{2 k_{\mathrm{F}}^{2} A}{C} .
$$

Here $\Gamma$ is the damping rate of coupled CDW and SDW fluctuation and $E^{*}$ is the order of the unrenormalized Fermi energy of band electrons.

Then, eq. (27) is written as

$$
\tilde{\epsilon}_{n}=\epsilon_{n}+\pi T \sum_{m=-\infty}^{\infty} M\left(\epsilon_{n}-\epsilon_{m}\right) \operatorname{sign}\left(\epsilon_{m}\right) .
$$

After some algebra, we can show that the left hand side of eq. (32) is reduced, in the limit of $T \rightarrow 0$ and up to the linear term of $\epsilon_{n}$, to

$$
\tilde{\epsilon}_{n}=\epsilon_{n}(1+\lambda) .
$$

Namely, $\lambda$ is the coupling constant giving the mass enhancement factor. Of course, there may exist other contributions to the mass enhancement arising from singlemode processes of exchanging spin and charge fluctuations with large nesting vector $\mathbf{Q}$. However, this contribution is expected to give only moderate enhancement for the Sommerfeld constant if the fluctuations are dominated by the nesting as has been discussed elsewhere. ${ }^{25}$ ) So, we neglect this contribution for simplicity of analysis.

In terms of $\lambda, \Gamma$ and $E^{*}$, the pairing interaction of p-wave $(\ell=1)$ is written as

$$
\begin{aligned}
& V\left(\ell=1, i \omega_{m}\right) \approx-\frac{\sqrt{3}}{N_{\mathrm{F}}} \frac{2 \lambda}{\ln \left|\frac{\Gamma+2 E^{*}}{\Gamma}\right|} \\
& \times\left[-1+\frac{E^{*}+\Gamma+\left|\omega_{m}\right|}{2 E^{*}} \ln \left|\frac{2 E^{*}+\Gamma+\left|\omega_{m}\right|}{\Gamma+\left|\omega_{m}\right|}\right|\right] .
\end{aligned}
$$

On the derivation above, the pairing interaction $V$ for a relevant channel can be expressed in a separable form in the wave vector and frequency space as

$$
V\left(\mathbf{k}-\mathbf{k}^{\prime}, \mathrm{i} \epsilon_{n}-\mathrm{i} \epsilon_{n^{\prime}}\right)=\phi(\mathbf{k}) \phi\left(\mathbf{k}^{\prime}\right) v\left(\mathrm{i} \epsilon_{n}-\mathrm{i} \epsilon_{n^{\prime}}\right),
$$

where $\phi(\mathbf{k})$ is the wavefunction of relevant pairing channel, and $v$ represents the frequency dependence of interaction due to the retardation. In the present case, $\phi(\mathbf{k}) \simeq$ $\sqrt{3} \hat{k}_{z}$, and $v$ is given by eq. (34). Thus, the gap function $\Delta$ also has the separable form $\Delta\left(\mathbf{k}, \mathrm{i} \epsilon_{n}\right)=\phi(\mathbf{k}) \Delta\left(\mathrm{i} \epsilon_{n}\right)$ and satisfies the gap equation

$$
\begin{aligned}
\Delta\left(\mathrm{i} \epsilon_{n}\right)=- & T \sum_{n^{\prime}} \sum_{\mathbf{k}^{\prime}} v\left(\mathrm{i} \epsilon_{n}-\mathrm{i} \epsilon_{n^{\prime}}\right) \\
& \times \frac{\left|\phi\left(\mathbf{k}^{\prime}\right)\right|^{2} \Delta\left(\mathrm{i} \epsilon_{n^{\prime}}\right)}{\xi_{\mathbf{k}^{\prime}}^{2}+\tilde{\epsilon}_{n^{\prime}}^{2}+\left|\phi\left(\mathbf{k}^{\prime}\right) \Delta\left(\mathrm{i} \epsilon_{n^{\prime}}\right)\right|^{2}},
\end{aligned}
$$

where $\xi_{\mathbf{k}}$ is the dispersion of quasiparticles without the renormalization due to the many-body effect of eq. (26), and $\tilde{\epsilon}_{n}$ is the renormalized frequency due to the manybody effect of eq. (27). Explicit form of $\tilde{\epsilon}_{n}$ in the present model is given by eq. (32).

The linearized gap equation is then given as follows:

$$
\Delta\left(\mathrm{i} \epsilon_{n}\right)=-T \sum_{n^{\prime}} v\left(\mathrm{i} \epsilon_{n}-\mathrm{i} \epsilon_{n^{\prime}}\right) \Delta\left(\mathrm{i} \epsilon_{n^{\prime}}\right) \sum_{\mathbf{k}^{\prime}} \frac{\left|\phi\left(\mathbf{k}^{\prime}\right)\right|^{2}}{\xi_{\mathbf{k}^{\prime}}^{2}+\tilde{\epsilon}_{n^{\prime}}^{2}} .
$$

At this stage, it may not be so bad to approximate $|\phi(\mathbf{k})|^{2}$ as an average $\left\langle|\phi(\mathbf{k})|^{2}\right\rangle$ near the Fermi level. This quantity is nearly equal to unity due to the normalization condition. Namely, eq. (37) can be approximated by the same one for the conventional s-wave pairing.

So far, we have constructed the general framework of the pairing mechanism mediated by coupled CDW and SDW fluctuations. To discuss the real materials rigorously, it is necessary to take into account of the band structure and the Fermi surface, accurately. Hereafter, we would like to discuss the anomalous behaviors of $H_{\mathrm{c} 2}(T)$ under pressures in $\mathrm{UGe}_{2}$. However, there is not enough information about the band structure and 
the Fermi surface responsible for the superconductivity under pressure and magnetic field in $\mathrm{UGe}_{2}$ at present. Hence, we proceed the discussion on the basis of the above framework with three-dimensional spherical band and consider the features of $\mathrm{UGe}_{2}$ by taking into account of the most-important factors such as pressure and magnetic-field dependences of mass-enhancement factor and $T_{\mathrm{x}}$ as will be discussed below. It is an important task to consider the accurate band structure and to determine the pairing symmetry of the orbital part in the next stage of the theoretical approach. However, we think that the anomalous behaviors of $H_{\mathrm{c} 2}$ under pressures do not depend on such details but are caused by the key factors which will be explained below.

Let us summarize again the behaviors of upper-critical field under pressures (see Fig. 2) and give its intuitive understanding. At $P=11.4 \mathrm{kbar}, H_{\mathrm{c} 2}$ shows only gradual increase in spite that $T_{\mathrm{SC}}$ was originally high at $H=0$. This is because $T_{\mathrm{x}}$, around which the attractive interaction is induced, goes up and the enhanced fluctuations associated with $T_{\mathrm{x}}$ fades away as $H$ increases. On the other hand, at $P=13.5 \mathrm{kbar} T_{\mathrm{SC}}$ is less than the maximum value at $H=0$. However, as $H$ increases, $T_{\mathrm{x}}$ goes up to be close and the attractive interaction is enhanced so that $H_{\mathrm{c} 2}$ grows rapidly. Then, $H_{\mathrm{c} 2}$ at $P=13.5 \mathrm{kbar}$ crosses above that of $P=11.4 \mathrm{kbar}$. As for $P=15.3$ kbar it is interpreted that $H_{\mathrm{c} 2}$ reaches $T=0$ before $T_{\mathrm{x}}$ closes up under magnetic field and thereby shows only the gradual increase.

The strong-coupling formalism for $H_{\mathrm{c} 2}$ originally developed for s-wave superconductors on the electronphonon mechanism, ${ }^{26,27)}$ can be extended rather easily for the present case. It is because the effect of magnetic field can be incorporated into the theory just as in the conventional case as far as the vector potential only couples with the center of mass coordinate of the wave function of the Cooper pair, but does not affect its relative coordinate. This is the case in usual situations since the size of the Cooper pair is far smaller than the width of the Landau orbital except for $H \simeq H_{\mathrm{c} 2}(T=0)$. Thus we can use the strong-coupling formalism for $H_{\mathrm{c} 2}$ developed for treating the s-wave pairing on a slight modification.

Starting from the Eliashberg equations, eq. (32) and eq. (36), one obtains the linearized gap equation under the magnetic field $H$ as follows:

$$
\tilde{\Delta}\left(i \epsilon_{n}\right)=\frac{T}{N_{\mathrm{F}}} \sum_{m} \tilde{V}\left(\epsilon_{n}-\epsilon_{m}\right) K\left(\tilde{\epsilon}_{m}\right) \tilde{\Delta}\left(i \epsilon_{m}\right),
$$

where the effective coupling $\tilde{V}$ is given by

$$
\tilde{V}\left(\omega_{m}\right)=-N_{\mathrm{F}} V\left(\ell=1, \mathrm{i} \omega_{m}\right)
$$

where $V\left(\ell=1, \mathrm{i} \omega_{m}\right)$ is defined by eq. (34). The integration kernel $K$ in eq. (38) is given as

$$
\begin{aligned}
K\left(\tilde{\epsilon}_{n}\right)= & \frac{4 \pi N_{\mathrm{F}}}{\sqrt{2 e v_{\mathrm{F}}^{2} H}} \int_{0}^{\infty} d q \exp \left(-q^{2}\right) \\
& \times \tan ^{-1}\left(\frac{q \sqrt{2 e v_{\mathrm{F}}^{2} H}}{2\left|\tilde{\epsilon}_{n}\right|}\right),
\end{aligned}
$$

where $e$ is the elementary charge, $v_{\mathrm{F}}$ is the Fermi velocity of band electrons and $\tilde{\epsilon}_{n}$ is defined by eq. (32). In the present case where only the electrons with majority spin participate in the formation of the SC state, the non-unitary triplet pairing is expected. Then the paramagnetic effect due to the Zeeman splitting has been neglected in deriving eq. (40). The strong-coupling effect is included in the frequency dependence of the gap function $\tilde{\Delta}\left(\mathrm{i} \epsilon_{n}\right)$ in eq. (38) and $\tilde{\epsilon}_{n}$ in eq. (40). The upper critical field $H_{\mathrm{c} 2}$ is determined by the condition that the linearized gap equation starts to have a nontrivial solution $\tilde{\Delta}\left(\mathrm{i} \epsilon_{n}\right) \neq 0$ as $H$ is decreased with $T$ fixed. The resultant equation to be solved is

$$
\operatorname{det}\left[\delta_{n m}-\frac{T}{N_{\mathrm{F}}} \tilde{V}\left(\epsilon_{n}-\epsilon_{m}\right) K\left(\tilde{\epsilon}_{m}\right)\right]=0,
$$

where $\delta_{n m}$ is the Kronecker delta symbol.

Our strategy for discussing $H_{\mathrm{c} 2}(T)$ is as follows: First, we determine the pressure dependence of $\lambda$ in eq. (28), giving the mass enhancement factor, by experimental data of specific heat. ${ }^{6)}$ Second, we estimate the pressure dependence of parameters $\Gamma$, eq. (30), and $E^{*}$, eq. (31), so as to reproduce $T_{\mathrm{SC}}$. Third, we deduce the magnetic-field dependence of the parameters with the use of a functional form of $T_{\mathrm{x}}(P, H)$ experimentally obtained. ${ }^{9,11)}$ Then, $H_{\mathrm{c} 2}(T)$ is calculated from eq. (41).

First of all, we discuss the $P$-dependence of $\lambda$ at $H=0$. In Fig. 8 open circles with an error bar denote the ratio of the specific-heat coefficient under finite and zero pressures, $\gamma(P) / \gamma(0)$, which are measured at $\left.H=0{ }^{6}\right)$ This ratio can be written in terms of the massenhancement factor as $\gamma(P) / \gamma(0)=[1+\lambda(P)] /[1+\lambda(0)]$. The enhancement of $\gamma(P)$ for $P \gtrsim 13 \mathrm{kbar}$ is considered to be contributed by two factors: one is the fluctuation associated with the anomaly at $T_{\mathrm{x}}$ and the other is the critical FM fluctuation near $T_{\mathrm{C}}$ which we do not consider here as irrelevant for the superconductivity. We consider the former is essential for inducing the SC state. Hence, we introduce a biquadratic function of $[1+\lambda(P)] /[1+\lambda(0)]$ around $P=P_{\mathrm{c}} \simeq 13 \mathrm{kbar}$ to fit $\gamma(P) / \gamma(0)$ as the dashed line in Fig. 8, although experimental data for $\gamma(P)$ remains to increase gradually even in the $P \gtrsim 13$ kbar region. We assume that the mass enhancement at zero pressure gives no contribution to the SC state for $10 \lesssim P \lesssim 17$ kbar, i.e., we set $\lambda(0)=0$. In this way, we now obtain the pressure dependence of the mass-enhancement factor, $\lambda(P)$ in eq. (28), which is denoted by the shaded line in Fig. 8 .

Secondly, we determine the $P$ dependence of $\Gamma$, eq. $(30)$, and $E^{*}$, eq. (31), so as to reproduce $T_{\mathrm{SC}}(P)$ at $H=0$ by using $\lambda(P)$ determined as above. In Fig. 8 open triangles denote 100 times of the SC transition temperature, i.e., $10^{2} T_{\mathrm{SC}}$. We assume the shape of $\Gamma(P)$ as the dash-dotted line in Fig. 8, which becomes narrow toward the critical pressure $P \sim 13.5 \mathrm{kbar}$. The open squares denote the values of $E^{*}$ which reproduce the experimental data of $T_{\mathrm{SC}}$ at each pressure. Here, we introduce a biquadratic function of $E^{*}(P)$ as the solid line in Fig. 8 to fit discrete data denoted by open squares. Then we obtain the $P$-dependence of $\lambda$, eq. (29), $\Gamma$, eq. (30), and $E^{*}$, eq. (31) from the experimental data of $\gamma$ and 


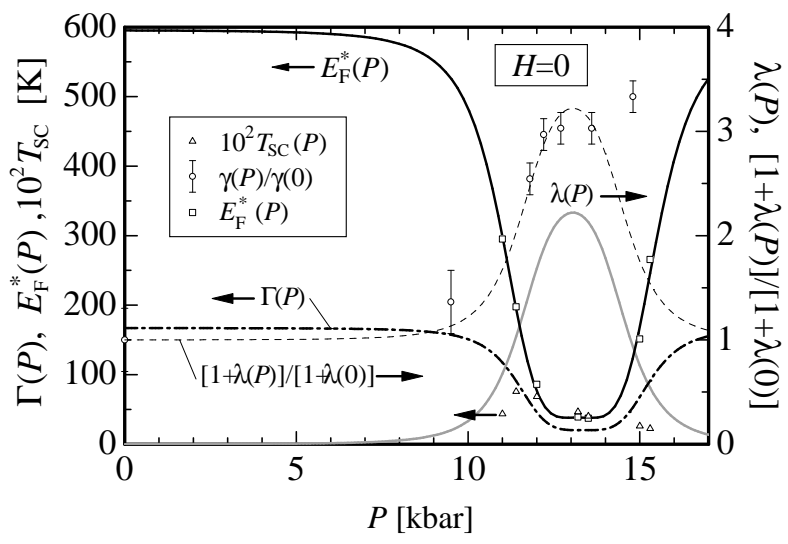

Fig. 8. Pressure dependence of parameters at $H=0$. Open circles with an error bar denote the ratio $\gamma(P) / \gamma(0)$ measured without magnetic field. ${ }^{6}$ ) Open triangles denote ten times of the SC transition temperature $T_{\mathrm{SC}} \cdot{ }^{2)}$ Open squares denote $E^{*}(P)$ which reproduce $T_{\mathrm{SC}}$ by using $\lambda(P)$ and $\Gamma(P)$. The dashed line and the shaded line denote $[1+\lambda(P)] /[1+\lambda(0)]$ and $\lambda(P)$, respectively. The dash-dot line and the solid line denote $\Gamma(P)$ and $E^{*}$, respectively.

$T_{\mathrm{SC}}$ in the $H=0$ case. If we choose another shape of $\Gamma(P)$, the shape of $E^{*}(P)$ also changes to reproduce $T_{\mathrm{SC}}$. However, the spectrum of coupled CDW and SDW fluctuations which mediate the superconductivity is considered to be sharp at $T_{\mathrm{x}}$ and thereby we here adopt the function $\Gamma(P)$ as shown in Fig. 8 .

Thirdly, we discuss the $H$-dependence of the parameters $\lambda, \Gamma$, and $E^{*}$. Here we note the $H$-dependence of $T_{\mathrm{x}}$ is identified by the rapid change of the slope of the resistivity $\rho(T)$ under the magnetic field at each pressure $^{9,28)}$ and is shown in Fig. 9(a). ${ }^{28)}$ We see that $T_{\mathrm{x}}$ depends on $H$ linearly at $P=11.4$ kbar and increases steeply at $P=13.5$ kbar. Incorporating these behaviors, we introduce a function $T_{\mathrm{x}}(P, H)$ as shown in Fig. 9(b). The key point is that $T_{\mathrm{x}}$ is an increasing function of $H$ and a decreasing function of $P$. By using this form of function $T_{\mathrm{x}}(P, H)$, the $H$ dependence of $\lambda$, eq. (29), and $\Gamma$, eq. (30), and $E^{*}$, eq. (31), is taken into account in the following way: We can obtain the pressure $\tilde{P}$ at $H=0$ which gives the same value of $T_{\mathrm{x}}$ at $P$ and $H$ by using the relation

$$
T_{\mathrm{x}}(\tilde{P}, H=0)=T_{\mathrm{x}}(P, H) .
$$

This always gives $\tilde{P} \leq P$ for $H \geq 0$. Namely, applying the positive magnetic-field turns out to be equivalent to the negative pressure at $H=0$. In this way we can take into account the effect of the magnetic field on parameters $\lambda, \Gamma$ and $E^{*}$ which appear in the gap equation eq. (41) of our model.

On these estimation of parameters, we can calculate $H_{\mathrm{c} 2}$ at each pressure as follows: The pressure $P$ and the magnetic field $H$ are fixed. With use of eq. (42), the reduced pressure $P=\tilde{P}$ is determined. Then, the linearized gap equation eq. (41), with parameters $\lambda(\tilde{P})$, $\Gamma(\tilde{P})$ and $E^{*}(\tilde{P})$ introduced in Fig. 8, is solved to obtain $H_{\mathrm{c} 2}$. The Fermi energy $\epsilon_{\mathrm{F}}$ and the Fermi velocity $v_{\mathrm{F}}$ of
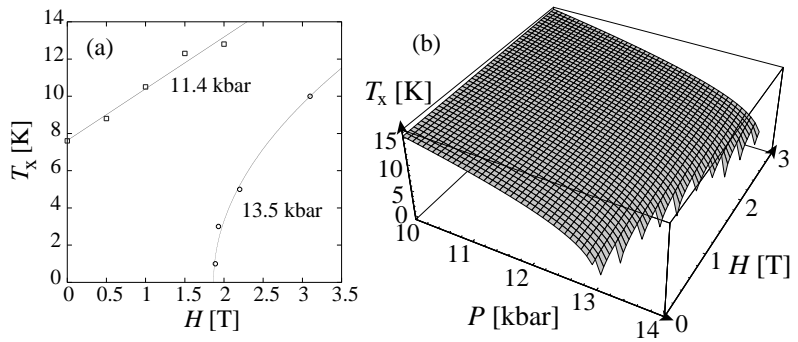

Fig. 9. (a) Experimental data of $T_{\mathrm{x}}$ as a function of filed applied parallel to the crystallographic a-direction at $P=11.4$ kbar and $P=13.5$ kbar. $^{28)}$ (b) Introduced function of $T_{\mathrm{x}}(P, H)$ to calculate $H_{\mathrm{c} 2}$ at each pressure.

unrenormalized band electrons have been set to reproduce the order of magnitude of observed $H_{\mathrm{c} 2}(T \rightarrow 0)$.

Results of $H_{\mathrm{c} 2}(T)$ for $P=11.4,13.5$ and 15.3 kbars are shown in Fig. 10. At $P=11.4$ kbar, where $T_{\mathrm{SC}}(H=0)$ is nearly maximum, $H_{\mathrm{c} 2}(T)$ merely increases gradually and monotonously as $T$ decreases. This is because applying the magnetic field gives negative pressure which makes $T_{\mathrm{SC}}(H)$ decreased, as seen in Fig. 8. This reflects the fact that $T_{\mathrm{x}}(H)$ goes up and away from $T_{\mathrm{SC}}(H=0)$ as $H$ increases at $P=11.4$ kbar. On the other hand, at $P=13.5 \mathrm{kbar}$, where $T_{\mathrm{SC}}(H=0)$ is rather smaller than the maximum at $P=11.4 \mathrm{kbar}, H_{\mathrm{c} 2}(T)$ increases steeply and shows first-order like "transition" at $H=1.8 \mathrm{~T}$. This anomalous increase is understood as a result of sudden appearance of $T_{\mathrm{x}}$ at $H=1.8 \mathrm{~T}$ due to the magnetic field as shown in Fig. 9(a). Alternatively, such an increase can be understood by the fact that the applied magnetic field has an effect of negative pressure which makes $T_{\mathrm{SC}}(H=$ 0 ) shift to the maximum value at $P=11.4$ kbar from $P=13.5$ kbar as shown in Fig. 8. From these results we can see that the crossing of $H_{\mathrm{c} 2}(T)$ at $P=11.4 \mathrm{kbar}$ and $P=13.5$ kbar is caused by two counter effects of the magnetic field: The suppression of $H_{\mathrm{c} 2}(H)$ at $P=$ 11.4 kbar and the enhancement of $H_{\mathrm{c} 2}(H)$ at $P=13.5$ kbar. At $P=15.3 \mathrm{kbar} H_{\mathrm{c} 2}$ shows the gradual and monotonous increase as $\mathrm{T}$ decreases. It is because the magnetic field $H \leq H_{\mathrm{c} 2}(T=0)$ is not enough to recover $T_{\mathrm{x}}(H)>0$ so that the concave $T$-dependence of $H_{\mathrm{c} 2}(T)$ is not realized.

If the $H$ dependence of $T_{\mathrm{x}}$ at $P=13.5$ kbar is gradual rather than the square-root-like behavior used in the present calculation as in Fig. 9(b) (for example, if $T_{\mathrm{x}}$ has a linear dependence with respect to $H$ ), the first-orderlike jump in $H_{\mathrm{c} 2}$ does not appear. However, $H_{\mathrm{c} 2}(T)$ still increases steeply and crosses that of $P=11.4 \mathrm{kbar}$. Then, the detail of the shape of $H_{\mathrm{c} 2}(T)$ changes according to the $H$ dependence of $T_{\mathrm{x}}$, but the global feature of $H_{\mathrm{c} 2}(T)$ under pressure such as crossing does not change.

The above result of $H_{\mathrm{c} 2}(T)$ is qualitatively the same as that obtained on a more simplified phenomenological model in which the effective coupling $\lambda\left(\omega_{m}\right)$, eq. (29), and the function for renormalization effect $M\left(\omega_{m}\right)$, 


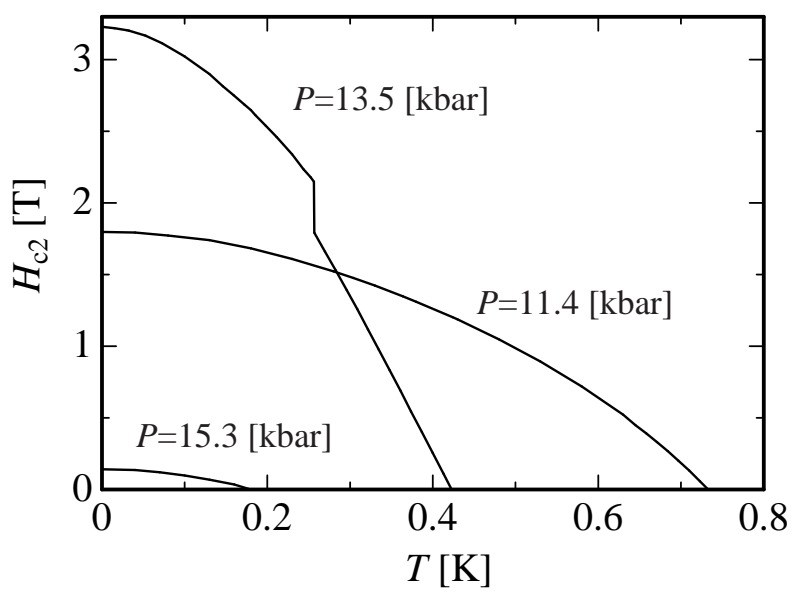

Fig. 10. The upper critical field $H_{\mathrm{c} 2}$ calculated based on the strong-coupling-superconductivity formalism explained in the text at $P=11.4$ kbar, 13.5 kbar and 15.3 kbar.

eq. (28), are approximated as

$$
\lambda\left(\omega_{m}\right) \approx M\left(\omega_{m}\right) \approx \frac{\lambda}{N_{\mathrm{F}}} \frac{\Gamma}{\Gamma+\left|\omega_{m}\right|} .
$$

As shown in ref, ${ }^{12,28)}$ this model explains the crossing of $H_{\mathrm{c} 2}(T)$ at $P=11.4$ and 13.5 kbars when the parameters $\lambda$ and $\Gamma$ are fixed in a way similar to the present paper.

\section{$\S 5$. Summary}

We have discussed that characteristic aspects of ferromagnetic superconductor $\mathrm{UGe}_{2}$ can be understood in a unified way on a single assumption that the superconductivity is mediated by the coupled CDW and SDW fluctuations originating from the CDW ordering of the majority-spin band at $T=T_{\mathrm{x}}$. The growth of extra magnetization below $T<T_{\mathrm{x}}$ is explained by a mode-coupling effect of the coupled CDW and SDW ordering caused by the imperfect nesting of the majority-spin band. The anomalously large entropy around $T=T_{\mathrm{x}}(P=0)$ is understood by the Kohn effect of softened optical phonons due to the onset of the CDW ordering of majority-spin band.

We have constructed the general framework of the pairing mechanism mediated by the coupled CDW and SDW fluctuations. The pairing interaction in the "p"wave channel among quasiparticles in the majority-spin band is shown to induce the ferromagnetic fluctuations enhanced around $P=P_{\mathrm{x}}$ due to the coupled CDW and SDW fluctuations. This mechanism also provides a natural explanation for the fact that the superconducting phase appears inside the ferromagnetic phase in the temperature-pressure $(T-P)$ phase diagram. The anomalous temperature dependence of the upper critical field $H_{\mathrm{c} 2}(T)$ observed at $P=11.4$ and $P=13.5$ kbars is understood by the strong-coupling formalism and the experimental fact that $T_{\mathrm{x}}$ is an increasing function of $H$.

Of course, there still remains many aspects to be clarified both experimentally and theoretically. A direct measurement of coupled CDW and SDW ordering at $T<T_{\mathrm{x}}$ is indispensable. In particular, the calculation of $\chi(\mathbf{q})$ based on the band-structure calculation and the search of CDW ordering by elastic neutron and X-ray scattering measurement is desired especially at around $P=9$ kbar where the decrease of $\rho(T)$ at $T=T_{\mathrm{x}}$ is much more prominent compared to the ambient pressure. Soft modes of phonons toward $T=T_{\mathrm{x}}$ should be observed at the wave vector $\mathbf{Q}$ where the nesting occurs. Origin of a first-order like ferromagnetic transition at $T=T_{\mathrm{C}}$ has not yet been clarified. The spontaneous magnetic moment, in the limit $T \rightarrow 0$, also exhibits a first-order like transition at $P=P_{\mathrm{x}} \cdot{ }^{8)}$ This is also to be clarified. The identification of the type of the superconducting order parameter is also important task left in the future. To identify the order parameter the group-theoretical classification is usually done. However, it is necessary to clarify the relevant effective interaction which works quasiparticles to identify the most stable order parameter among the possible candidates. ${ }^{4,5)} \mathrm{We}$ suppose that the superconducting gap opens at the parts of the Fermi surface with crystalline inversion symmetry, which is different from those with the CDW gap to gain both the condensation energies of CDW and superconducting states. The steep decrease of the superconducting temperature, $T_{\mathrm{SC}}$ for the $P<P_{\mathrm{x}}$ region in the $T$ - $P$ phase diagram may be due to the partial collapse of the Fermi surface by the imperfect nesting.

Quite recently, it has been discovered that ferromagnetic metal URhGe, at $T<10 \mathrm{~K}$, sets in superconducting state at $T \simeq 0.35 \mathrm{~K}$ under the ambient pressure. ${ }^{29)}$ This compound seems to share common aspects with $\mathrm{UGe}_{2}$, such as the crystal structure with zigzag chain of $\mathrm{U}$ atoms, existence of a small hump of $C / T$ at $T \sim T_{\mathrm{C}} / 3$ as seen in Fig. 6, enhanced ratio $A / \gamma^{2}$ of 10 times larger than the so-called Kadowaki-Woods ratio, ${ }^{30)}$ suggesting that the system is located near the quantum critical point in a kind of another, and of course the coexistence of superconductivity and ferromagnetism. The experimental evidence of the CDW has not been reported in URhGe at present. However, we expect that the small hump of $C / T$ at $T \sim T_{\mathrm{C}} / 3$ may be the signature of the CDW transition, since the zig-zag-chain structure of $U$ atoms, which is a common feature of $\alpha$-Uranium, $\mathrm{UGe}_{2}$ and $\mathrm{URhGe}_{2}$, provides the low dimensionality of the Fermi surface which enlarges the nesting instability. Further studies of URhGe as well as $\mathrm{UGe}_{2}$ are expected to extend the frontier of a class of ferromagnetic superconductor.

\section{Acknowledgments}

This work started from conversations with members of Flouquet group at DRFMC of CEA/Grenoble when one of us (K.M.) was staying there. We have much benefited from discussions with A. Huxley, I. Sheikin, D. Braithwaite, and J. Flouquet. Unpublished experimental data they showed us prior to publication was essential for us to develop a theoretical idea. We acknowledge $\mathrm{N}$. Tateiwa, T. C. Kobayashi, and Y. Onuki for allowing us to use their experimental data of specific heat as well as fruitful discussions. We also acknowledge H. Yamagami for providing his result of band structure calculations prior to publication as well as valuable discussions. K.M. has benefited from conversations with G. 
Lonzarich, V. P. Mineev, and C. Pfleiderer on the pairing interaction, which led us to clarify the presentations in $\S 4$. S.W. thanks M. Sigrist for useful discussion. Thanks are also due to D. Aoki, K. Hori, G. Oomi, N. K. Sato, R. Settai and H. Kohno for useful discussions. This work was supported by the Grant-in-Aid for COE Research (No.10CE2004) from Monbu-Kagaku-sho, and in part by a Grant-in-Aid for Scientific Research for Specified Area (No.12046246) by the Japan Society for Promotion of Science.

1) S. S. Saxena, P. Agarwal, K. Ahilan, F. M. Grosche, R. K. W. Hasselwimmer, M. J. Steiner, E. Pugh, I. R. Walker, S. R. Julian, P. Monthoux, G. G. Lonzarich, A. Huxley, I. Sheikin, D. Braithwaite and J. FLouquet: Nature 406 (2000) 587.

2) A. Huxley, I. Sheikin, E. Ressouche, N. Kernavanois, D. Braithwaite, R. Calemczuk and J. Flouquet: Phys. Rev. B 63 (2001) 144519.

3) A. M. Clogston: Phys. Rev. Lett. 9 (1962) 266.

4) K. Machida and T. Ohmi: Phys. Rev. Lett. 86 (2001) 850.

5) I. A. Fomin: JETP Lett. 74 (2001) 111.

6) N. Tateiwa, T. C. Kobayashi, K. Hanazono, K. Amaya, Y. Haga, R. Settai and Y. Ōnuki: J. Phys. C. 13 (2001) L17.

7) G. Oomi, K. Nishimura, T. Kagayama, S. W. Yun and Y. Onunki: Physica B 186-188 (1993) 758.

8) A. Huxley, private communications.

9) N. Tateiwa, K. Hanazono, T. C. Kobayashi, K. Amaya, T. Inoue, K. Kindo, Y. Koike, N. Metoki, Y. Haga, R. Settai and Y. Ōnuki: J. Phys. Soc. Jpn. 70 (2001) 2876.

10) G. Motoyama, S. Nakamura, H. Kadoya, T. Nishioka and N. K. Sato: Phys. Rev. B, in press.

11) I. Sheikin, private communications.

12) I. Sheikin, A. Huxley, D. Braithwaite, J.-P. Brison, S. Watanabe, K. Miyake and J. Flouquet: Phys. Rev. B 64220503 (2001).

13) H. Yamagami and A. Hasegawa: Physica B 186-8 (1993) 182; S. Tejima, H. Yamagammi and N. Hamada: preprint, May 2000.

14) A. B. Shick and W. E. Pickett: Phys. Rev. Lett. 86 (2001) 300.

15) H. Yamagami, private communications.

16) J. C. Marmeggi, R. Currat, A. Bouvet and G. H. Lander: Physica B 263-4 (1999) 624.

17) M. Naito and S. Tanaka: J. Phys. Soc. Jpn. 51 (1982) 219.

18) R. Settai, M. Nakashima, S. Araki, Y. Haga, T. C. Kobayashi, N. Tateiwa, H. Yamagami and Y. Ōnuki: J. Phys.: Condens. Matter 14 (2002) L29.

19) N. Suzuki and K. Motizuki: in Structural Phase Transition in Layered Transition-Metal Compounds, edited by K. Motizuki (Kluwer Academic Publishers, Dordrecht, 1986), P. 135.

20) T. Matsuura, K. Miyake, H. Jichu, Y. Kuroda and Y. Nagaoka: J. Magn. Magn. Matter. 52 (1985) 239.

21) M. T. Béal-Monod, C. Bourbonnais and V. J. Emery: Phys. Rev. B 34 (1986) 7716.

22) K. Miyake, S. Schmitt-Rink and C. M. Varma: Phys. Rev. B 34 (1986) 6554

23) D. J. Scalapino, E. Loh, Jr. and J. E. Hirsch: Phys. Rev. B 34 (1986) 8190.

24) A. J. Leggett: Phys. Rev. 140 (1965) A1869.

25) H. Maebashi and K. Miyake: Physica B 281\&282 (2000) 526; K. Miyake and H. Maebashi: J. Phys. Chem. Solids 62 (2001) 53.

26) E. Felfand and N. R. Werthamer: Phys. Rev. Lett. 13 (1964) 686; Phys. Rev. B 147 (1966) 288.

27) M. Schossmann and E. Schachinger: Phys. Rev. B 33 (1986) 6123.

28) J. Flouquet, A. Huxley, I. Sheikin, N. Kernavanois, D. Braithwaite, E. Ressouche, S. Watanabe and K. Miyake: J. Phys. Soc. Jpn. 70 (2001) Suppl. 14.

29) D. Aoki, A. Huxley, E. Ressouche, D. Braithwaite, J. Flou- quet, J.-P. Brison, E. Lhotel, C. Paulsen: Nature 413 (2001) 613.

30) D. Aoki: private communications. 